Interestingly, this protein was found to be a ligand-gated channel ${ }^{5}$, not a GPCR. Other groups have identified whole families of GPCRs related to olfactory, or peptide receptors, and present in either the lingual epithelium or the taste buds $^{6,7}$. But no ligands to any of these cloned receptors have been identified. The most promising approach taken so far characterized a newly discovered variant of a metabotropic glutamate receptor $^{8,9}$. This truncated mGluR4, the 'umami' receptor, is the first taste-specific GPCR cloned and characterized with respect to ligand. It is the taste receptor for monosodium glutamate, a key ingredient of Chinese food.

To determine their ligand specificity, cloned GPCRs can be expressed in a cell line or in oocytes. This approach requires that the protein avoid pre-Golgi degradation in the 'foreign' cell, be able to reach the cell surface and be correctly folded for binding its ligand and functionally couple to those $\mathrm{G}$ proteins and transduction cascades present. These complex requirements may explain why the literature reports many GPCRs for which a ligand cannot be specified.

Because Hoon's team also had difficulty with this direct, molecular, means of finding the ligand to their receptors, they used 'topographic selectivity'. Here sensory areas of the mouth are taken to have taste preferences. Thus, by noting in which area the receptor is expressed, one can guess at the taste quality de- tected. However, their use of taste maps is not without problems. Maps such as that presented in Fig. 1 of their paper (and found in many text books) are easily misinterpreted. The areas shown are taken to be areas of 'preference' even though some 'overlap' is conceded. But the original reports-seldom quoted with the diagrams-say otherwise. A careful study by Hänig ${ }^{10}$, the earliest source of human tongue maps, reports differences in threshold between areas of the tongue (and the palate) for three of four taste qualities, but 100\% "overlap" (see Fig.). The detection threshold for sucrose, for instance, was determined to 10 $\mathrm{mM}$ at the tip of the tongue and $35 \mathrm{mM}$ in the back. This difference is not large when compared to the concentration range in which the intensity of sucrose taste increases. Preference, which would require a comparison of intensities across taste qualities, was not determined. Further references on chemotopic maps can be found at www. med-rz.uni-sb.de/med_fak/physiol2/LDM/ index.htm.

However, the human taste map does not apply to Hoons' data from rats and mice. The rat, unlike humans (and hamsters), is known to show a larger response when sucrose is used to stimulate the palate or the posterior tongue, compared with stimulation of the anterior tongue ${ }^{11}$. Thus, unfortunately, the expression pattern observed for TR1, although interesting in itself $_{\iota}$ does not match the expected pattern for sweet taste in the rat. Therefore, the race continues to clone the sweet receptor.

1. Hoon, M.A. et al. Putative mammalian taste receptors: A class of taste specific GPCRs with distinct topographic selectivity. Cell 96, 541-551 (1999).

2. Wong, G.T., Gannon, K.S. \& Margolskee, R.F. Transduction of bitter and sweet taste by gustducin. Nature 381, 796-800 (1996).

3. Lindemann, B. Taste reception. Physiol. Rev. 76, 719-766 (1996).

4. Ozaki, M., Amakawa, T., Ozaki, K. \& Tokunaga, F. Two types of sugar-binding protein in the labellum of the fly - putative taste receptor molecules for sweetness. J. Gen. Physiol. 102, 201-216 (1993).

5. Grosvenor, W. et al. The arginine taste receptor. Physiology, biochemistry, and immunohistochemistry. Ann NY Acad. Sci. 855, 134-142 (1998).

6. Abe, K., Kusakabe, Y., Tanemura, K., Emori, Y. \& Arai, S. Primary structure and cell-type specific expression of a gustatory $\mathrm{G}$ protein-coupled receptor related to olfactory receptors. J. Biol. Chem. 268, 12033-12039 (1993).

7. Matsuoka, I., Mori, T., Aoki, J., Sato, T. \& Kurihara, K. Identification of novel members of G-protein coupled receptor superfamily expressed in bovine taste tissue. Biochem. Biophys. Res. Comm.194, 504-511 (1993).

8. Chaudhari, $\mathrm{N}$. et al. The Taste of Monosodium Glutamate: Membrane receptors in taste buds. J. Neurosci. 16, 3817-3826 (1996).

9. Landin, A.M. \& Chaudhari, N. Functional expression of brain- and taste-specific forms of metabotropic glutamate receptor 4 (mGluR4) in cultured Chinese Hamster Ovary (CHO) cells. Chem. Senses (in the press).

10. Hänig, D.P. Zur Psychophysik des Geschmackssinnes. Philosophische Studien 17, 576-623 (1901).

11. Smith, D.V. \& Frank, M.E. Sensory coding by peripheral taste fibers. in Chapter 12: M echanisms of Taste Transduction (eds. Simon, S.A. \& Roper, S.D.) 295-338 (CRC Press, Boca Raton, 1993).

Department of Physiology

BIdg 58, Saar University, D- 66421

Homburg, Germany

e-mail phblin@med-rz.uni-sb.de

\title{
The invasive side of cyclosporin
}

Transplant patients are often given the immunosuppressant drug cyclosporin A to prevent graft rejection. The increased occurrence of cancer in these patients was believed to be due to the inability of the suppressed immune system to eliminate cancerous cells. In a study published in the 11 Feb 1999 issue of Nature, Hojo et al. demonstrated that cyclosporin A actually promotes tumor progression directly, independently of its effects on the host immune system.

Treatment of cells in culture with cyclosporin A caused phenotypic alterations characteristic of invasive cells, such as membrane ruffling and formation of numerous pseudopodia. Furthermore, after injection of murine adenocarcinoma cells into SCID-beige mice, treatment

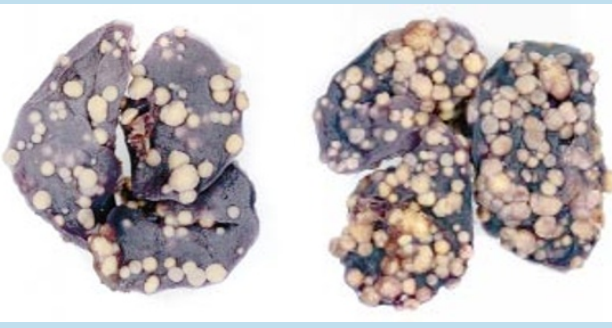

with cyclosporin A resulted in an increased number of metastases. Cyclosporin A increases the number of pulmonary metastases, as clearly shown in photographs of lungs taken from untreated mice (left) and from cyclosporintreated mice (right).

"We have identified a potential mechanism for the well-known increase in metastatic cancers in transplant patients," said senior author Manikkam
Suthanthiran. "These findings make sense, given previous reports that cyclosporin A increases expression of TGF- $\beta$ in several cell types, and that TGF- $\beta$ production promotes tumor cell invasion and metastasis." Suthanthiran also added that the findings do not mean that cyclosporin A causes cancer, but that if patients taking cyclosporin A already have cancer, the cancer is more likely to be invasive.

The findings suggest a possible therapeutic target pathway for patients who receive organ transplants and for other individuals at increased risk for neoplasms. Suthanthiran plans to continue these studies on other agents known to increase TGF- $\beta$ expression and on other immunosuppresive drugs such as FK506. Kristine N ovak, N ew York 